\title{
O ADOLESCENTE E SUA SEXUALIDADE: UMA ABORDAGEM EM EDUCAÇÃO E SAÚDE NA ESCOLA
}

Adriana Leal Moraes ${ }^{1}$, Sandra Cristina dos Santos Costa ${ }^{1}$, Selma Silva da Silva1, Margarete Feio Boulhosa ${ }^{2}$, Elisa da Silva Feitosa², Carla Monique Lavareda Costa ${ }^{3}$

Objetivo: Analisar o conhecimento de estudantes sobre sexualidade e seus riscos de adquirir infecções sexualmente transmissiveis. Metodologia: o estudo é do tipo descritivo, exploratório de caráter qualitativo, ocorreu em uma escola particular de ensino fundamental em Belém-PA no periodo de setembro a outubro de 2015. Participaram 15 adolescentes na faixa etária de 14-16 anos. Os dados foram coletados por meio de entrevistas semiestruturadas gravadas, com um roteiro sistematizado, e foram submetidos à análise de conteúdo. Resultados: A maioria dos adolescentes era do sexo masculino, com idade predominante de 14 anos. A análise evidenciou quatro categorias. Conclusões: percebeu-se um déficit no conhecimento sobre a sexualidade. O pouco conhecimento que os adolescentes tem os expõe a riscos. Nesse contexto, é necessário que os profissionais abordem, no ambiente escolar, sobre a vida sexual desses indivíduos envolvendo o seio familiar nesse processo de educação em saúde.

Descritores: Adolescente; sexualidade; Sducação em saúde; Doenças Sexualmente Transmissíveis.

\section{TEENAGER AND HIS SEXUALITY: AN APPROACH ON EDUCATION AND HEALTH IN SCHOOL}

Objective: To analyze students' knowledge about sexuality and its risks of acquiring sexually transmitted infections. Methodology: the descriptive, exploratory, qualitative study was carried out at a private elementary school in Belém-PA from September to October 2015. Fifteen adolescents in the 14-16 age group participated. The data were collected through semistructured recorded interviews, with a systematized script, and were submitted, according to content analysis. Results: The majority of adolescents were male, with a predominant age of 14 years. The analysis revealed four categories. Conclusions: There was a deficit in knowledge about sexuality. The little knowledge that adolescents have exposes them to risks. In this context, it is necessary for professionals to approach, in the school environment, the sexual life of these individuals involving the family in the process of health education.

Descriptors: Adolescent; Sexuality; Health education; Sexually Transmitted Diseases.

\section{ADOLESCENTE Y SU SEXUALIDAD: UN ENFOQUE EN LA EDUCACIÓN Y LA SALUD EN LA ESCUELA}

Objetivo: Analizar el conocimiento de los estudiantes sobre sexualidad y sus riesgos de adquirir infecciones de transmisión sexual. Metodología: el estudio es del tipo descriptivo, exploratorio de carácter cualitativo, ocurrió en una escuela particular de enseñanza fundamental en Belém-PA en el período de septiembre a octubre de 2015. Participaron 15 adolescentes en el grupo de edad de 14-16 años. Los datos fueron recolectados por medio de entrevistas semiestructuradas grabadas, con un itinerario sistematizado, y fueron sometidos al análisis de contenido. Resultados: La mayoría de los adolescentes era de sexo masculino, con edad predominante de 14 años. El análisis mostró cuatro categorías. Conclusiones: se percibió un déficit en el conocimiento sobre la sexualidad. El poco conocimiento que los adolescentes tienen los expone a riesgos. En este contexto, es necesario que los profesionales aborden, en el ambiente escolar, sobre la vida sexual de esos individuos involucrando el seno familiar en ese proceso de educación en salud.

Descriptores: Adolescente; sexualidad; Educación en salud; Enfermedades de transmisión sexual. 


\section{INTRODUÇÃO}

O Estatuto da Criança e do Adolescente, Lei no 8.069/1990 art. 2 ㅇ considera adolescente aqueles indivíduos com faixa etária entre 12 e 18 anos de idade. Já para a Organização Mundial de Saúde considera a idade entre 10 e 19 anos incompletos ${ }^{(1)}$. Sabe-se que a sexualidade é parte integral do desenvolvimento humano, sendo de fundamental importância o apoio ao adolescente no esclarecimento de possíveis dúvidas. Nesse contexto, a escola tem um papel importante na formação do indivíduo e representa um espaço propício para trabalhar competências, conhecimentos e mudanças de comportamentos, uma vez que é o lugar onde o adolescente permanece o maior tempo de seu dia ${ }^{(2)}$.

No Brasil, a temática da sexualidade vem ganhando espaço nas escolas com o objetivo principal de prevenir Infecções Sexualmente Transmissiveis (IST) e gravidez na adolescência $^{(3)}$. A percepção dos riscos de adquirir uma IST varia de pessoa para pessoa, e sofre mudanças ao longo da vida. Observa-se que os pais/responsáveis e a equipe de saúde, comumente, tendem a não abordar aspectos determinantes da saúde sexual dos adolescentes, devido à negação do desejo sexual do jovem e ao incentivo ao prolongamento da infância(4).

Para combater as IST, é importante a realização de ações de saúde que interrompam a cadeia de transmissão, detectando precocemente os casos, tratando os infectados e seus parceiros, prevenindo-os de outras IST e de complicações advindas das infecções, a partir do aconselhamento(5).

Frente à temática surgiu à preocupação de como as informações sobre sexualidade estão sendo entendidas por esses adolescentes?

O tema escolhido é de suma importância quanto aos aspectos biológico e psicossociais do adolescente, levando em consideração as informações a respeito da sexualidade na adolescência. Visando desenvolver também conhecimento técnico-científico e habilidades como forma de aprofundar a nossa experiência sobre a temática.

Sendo assim esta pesquisa tem o objetivo de analisar o entendimento dos adolescentes sobre sexualidade a partir do recebimento de informações oriundas tanto dos professores da escola como da família.

\section{METODOLOGIA}

\section{Tipo de estudo}

Realizou-se uma pesquisa descritiva, exploratória de caráter qualitativo. Por se tratar de uma metodologia que requer a participação ativa do sujeito de pesquisa. Na descrição as opiniões dos informantes são apresentadas da maneira mais fiel possivel, como se os dados falassem por si próprios ${ }^{(6)}$. Assim, a pesquisa qualitativa responde a questões muito particulares. Ou seja, ela trabalha com o universo dos significados, dos motivos, das aspirações, das crenças, dos valores e das atitudes. Esse conjunto de fenômenos humanos é entendido aqui como parte da realidade social, pois o ser humano se distingue não só por agir, mas pensar sobre o que faz e por interpretar suas ações dentro e a partir da realidade vivida e partilha com seus semelhantes ${ }^{(6)}$.

\section{Participantes da pesquisa}

A população da pesquisa foi composta por adolescentes de ambos os sexos, faixa etária de 14 a 16 anos de idade, sendo a maioria com idade igual a 14 anos, matriculados no nivel fundamental de ensino, com renda familiar mensal de cerca de dois salários mínimos.

\section{Local do estudo}

O estudo ocorreu em uma Escola Privada de ensino fundamental, na área metropolitana de Belém/PA nos turnos manhã e tarde, no período de setembro a outubro de 2015. Os participantes da pesquisa foram adolescentes devidamente matriculados, pertencentes a faixa etária entre 14 a 16 anos, que estiveram cursando da 6a a $8^{a}$ série do ensino fundamental de forma assidua.

\section{Procedimentos de coleta de dados}

Foram aplicados dois questionários com o uso de um gravador portátil, sendo o primeiro referente à caracterização da população do estudo e o segundo acerca da percepção do adolescente sobre sexualidade após recebimento de informação tanto na escola como no seio familiar. O local utilizado para a coleta dos dados foi uma das salas de aula disponibilizada pela instituição e, para assegurar o sigilo, foi utilizada a letra "A" de adolescente seguindo de sequência numérica a cada entrevistado. Dessa forma a transcrição das falas foi a mais fidedigna e o sigilo preservado.

\section{Procedimentos de análise dos dados}

Após a transcrição dos dados colhidos, a análise de conteúdo foi empregada. Na análise o propósito é ir além do descrito, fazendo uma decomposição dos dados e buscando as relações entre partes que foram decompostas e, por último, na interpretação - que pode ser feita após a análise ou após a descrição - busca-se sentido das falas e das ações para se chegar a uma compreensão ou explicação que vão além do descrito e analisado(b). 


\section{Procedimentos éticos}

O estudo foi aprovado pelo Comitê de Ética em Pesquisa com Seres Humanos da Fundação Pública Estadual Hospital de Clinicas Gaspar Viana (FHCGV), e está de acordo com a Resolução 466/12 do Conselho Nacional de Saúde com o parecer de número: 1.486.219.

\section{RESULTADOS}

Os adolescentes participantes do estudo foram identificados pela letra A de "adolescente" seguidos por números arábicos, mantendo-se a ordem de realização das entrevistas: Al a Al5. Dos informantes, todos pertenciam a faixa etária de 14 e 16 anos de idade, sendo onze de 14 anos, três de 15 anos e apenas um de 16 anos. Quanto ao sexo predominava o masculino em número de oito, e sete do sexo feminino. Com relação a moradia 14 adolescentes moram em casa própria e um com parentes. Quanto a religião nove são católicos, cinco são evangélicos e uma se autodenomina em ambas em respeito aos pais.

Quanto a série escolar nove são da 7ạ série e seis são da 8a série. Já quanto ao turno em que estudam oito são do turno vespertino e sete do turno matutino. Em relação a renda familiar sete recebem (02) dois salários mensais, (01) um recebe quatro salários mensais, (01) um recebe seis salários mensais e (06) seis adolescentes não quiseram relatar. Quanto ao responsável principal, (06) seis moram com os pais, (5) cinco apenas com a mãe, (03) três moram com o pai e apenas (Ol) um mora com a mãe/vó.

A partir da análise dos dados, emergiram 04 (quatro) categorias temáticas e suas respectivas sub categorias.

\section{Visão do adolescente sobre conceitos de adolescência}

Por meio dos relatos dos informantes, foi possivel observar o que representa adolescência para os adolescentes em seu sentido amplo, incluida e externando-se a escola. Esse entendimento variou nas falas, sendo relatados por meio do conhecimento e experiências de vida que os mesmos possuíam.

\section{Adolescência e puberdade}

Os relatos abaixo abordaram adolescência como descobertas de novos conhecimentos sobre si, evidenciado nas falas: $E$ quando deixamos de ser crianças [...] é quando a gente ganha responsabilidade (A9). Acho que é uma fase de maturidade, uma fase de esperas (A4). Infere-se também que percebem ser o início da adolescência marcado pela puberdade: É uma época de várias descobertas e mudanças (A6). Um caminho para o futuro [...] (A2).

\section{Sexualidade e seu conhecimento pelo adolescente}

Após as colocações do sentido da palavra adolescência, os adolescentes foram questionados quanto a aplicação deste termo no contexto escolar, para que de forma indireta, pudessem construir um conceito para educação em saúde sexual com ênfase para a fase da puberdade em que se encontram, sem, contudo, o pesquisador tê-la pronunciado durante a entrevista.

\section{Debatendo sexualidade na escola}

Desse modo, infere-se das falas que entendem ser a sexualidade o desenvolvimento do corpo e suas características tanto físicas como emocionais. Assim, os conceitos elaborados por eles se apresentaram como: Diferença do sexo para outro (A5). Ele fala que é o sexo de ambos mulher e homem (A2). A sexualidade é diferente de sexo, é um relacionamento entre homem e mulher (A8). Outro aspecto evidenciado também foi de alguns participantes da pesquisa fazerem a correlação de sexualidade com opção sexual. Quando me falam sobre sexualidade, se a pessoa é homossexual, bissexual (A6). Na minha opinião também e a escolha do sexo da pessoa tem muitos pais que não aceitam a escolha do filho ou da filha... (A12). Dos quinze participantes da pesquisa três destacaram algum conhecimento relacionado à questão colocada a eles. $\dot{E}$ o conhecimento do meu próprio corpo (A15). É uma coisa assim, tu aprende um pouco do teu corpo, como ele é como tu deve te comportar, como tu deve te sentar direito, tu deve ficar como um moça (A5). As mudanças do corpo (A7). Houve quem dissesse que desconhecia o assunto por completo: Nunca nem ouvi falar disso ai (A4).

\section{Infecções Sexualmente Transmissiveis (IST's) e adolescência}

Foi possivel identificar o conhecimento dos adolescentes acerca das IST's, ora ensinado em casa ora na escola. Assim, percebe-se que foi repassada alguma orientação sobre o assunto frente aos cuidados preventivos e acerca do autocuidado, essencial para manutenção da saúde. No que se refere ao conhecimento deles em relação a IST (Infecções sexualmente transmissiveis), observamos que esse assunto não é totalmente desconhecido pelos participantes da pesquisa. Mas que devido ao déficit de informação relacionada ao desenvolvimento da sexualidade faz com que fiquem mais expostos aos riscos. 


\section{Nivel de informação acerca das IST's entre os adolescentes}

Nesta subcategoria constatou-se os conhecimentos prévios recebidos pelos adolescentes. Desta maneira, observouse relatos de conhecimento sobre a transmissão de doenças por via sexual: [...] ela transmite uma doença muito séria, tem que ter muito cuidado [...] (A5). Pessoas que se relacionam com a outra sem proteção, e se infectam por essas doenças, praticam sexo sem proteção (A8). Já outros participantes apenas falam que conhecem, mas não explicam o que vem a ser IST, mas que receberam informação na escola com o professor. Eu entendo sobre AIDS e várias outras conheci na escola (A14). Sifilis. Recebi informação pelo professor (A12). Já outros tiveram informação tanto na escola como pelos pais, mais com as mães falam ser mais amigas. Mas com a minha mãe tenho mais liberdade e nós conversamos muito com ela é muito minha amiga, ai depois foi na escola começaram a fazer palestras sobre doenças e como prevenir (A4). Teve uma palestra na minha escola e também minha familia conversou comigo sobre o assunto (A6).

\section{Discutindo Sexualidade na escola}

Constatou-se que os conhecimentos sobre saúde sexual entre os adolescentes existem, mas que não são tão completos como deveria, sendo necessário pensar em novas estratégias para que o conhecimento sobre o assunto somado a prevenção de riscos relacionados a IST's seja mais eficaz.

\section{Discutindo estratégias educacionais para abordagem da saúde sexual nas escolas}

Evidenciou-se sugestões de estratégias a serem adotadas por quem transmite as informações para que o recebimento das mesmas seja eficaz. Através de palestra sobre saúde, e conversa com a familia, mais os colegas também ajudam de certa forma nesse assunto [...] (A6). Pelos professores porque as vezes conversa, mas com a gente (A9). Destaca-se o diálogo como forma de desenvolver estratégias para se abordar a temática com os adolescentes de forma clara, mas com discussões sobre o assunto em questão. No entanto surge através das falas 1 (um) dos adolescentes, que diz que prefere conversar com um amigo por ser sentir mais à vontade: Colegas porque eu me sinto mais solto, consigo relatar tudo detalhe por detalhe (A12). E os que preferem não dialogar com ninguém e procuram informação nos meios de comunicação. Acho melhor, tenho vergonha de perguntar para minha mãe e na internet acho tudo (A15).

\section{DISCUSSÃO}

A adolescência consiste no período de transição entre a infância e a vida adulta, caracterizando-se pelos impulsos do desenvolvimento biológico, psicológico e social. Essa fase se inicia com as mudanças corporais da puberdade, caracterizando-se pelo despertar da sexualidade, desenvolvimento da personalidade, além de esforços para integração em grupos sociais ${ }^{(7)}$. Assim, o conhecimento do adolescente sobre adolescência influencia positivamente a vivência desta fase da vida.

$O$ adolescente tem direito a uma vida sexual e deve tomar suas próprias decisões, conscientemente, baseadas no conhecimento. Para que isso aconteça, entretanto, é necessário que os profissionais de saúde assistam a esse público de acordo com suas características, garantindolhes condições de acesso aos serviços de saúde, educação em saúde e contracepção(8).

Apesar de identificar nas entrevistas o pouco conhecimento sobre o significado da sexualidade, os adolescentes identificam as transformações pelas quais estão passando em relação ao desenvolvimento e crescimento desta fase.

A sexualidade manifesta-se no ser humano como um fenômeno biológico, psicológico e social que influencia o seu modo de estar, compreender e viver o mundo como ser sexuado: homem e mulher. É um fenômeno plural que não é e não está dado apenas pela natureza. Os comportamentos e as práticas sexuais, os sentimentos e os desejos estão inseridos e são influenciados pela forma como as sociedades se organizam e pelas relações estabelecidas entre as pessoas que dela fazem parte ${ }^{(9)}$.

Assim, a maneira como os e as adolescentes vão expressá-la e vivê-la é influenciada por vários fatores entre os quais estão: a qualidade das relações, emocional e afetiva, que viveram com as pessoas significativas na infância e na sua vivência atual; relações com seus grupos de pares; as transformações físicas, psicológicas, cognitivas e sociais trazidas pelo crescimento e desenvolvimento, até os valores, crenças, normas morais e tradições da família e da sociedade na qual eles e elas estão inseridos(as) ${ }^{(9)}$.

Há adolescentes que redirecionam o conceito de sexualidade para a simples escolha da opção sexual a ser seguida, e há também os que digam nunca terem recebido informação a respeito.

Nesse cenário, é fundamental que os serviços de saúde ampliem a cobertura e facilitem o acesso de adolescentes homens e mulheres às unidades de saúde e que as equipes de profissionais reconheçam a importância do seu papel no cuidado diferenciado a essas pessoas como sujeitos de direitos, o que requer sensibilidade e disponibilidade para exercerem sua prática procurando a melhoria da qualidade no atendimento(9).

Embora haja adolescentes com algum conhecimento sobre Infecções Sexualmente Transmissiveis, ainda é um

152 I Enferm Foco [Internet]. 2019;10(2): 149-154. 
assunto que deve ser abordado com maior ênfase nas escolas e no seio familiar.

No mundo todo, em cada 20 adolescentes, um contrai algum tipo de DST anualmente ${ }^{(10)}$. Nesse contexto, observase que os adolescentes engajam-se cada vez mais em comportamentos de risco, que podem trazer consequências negativas, a curto e longo prazo. Entre eles, está a prática do sexo sem proteção. Várias são as razões do comportamento sexual desprotegido entre adolescentes e, dentre elas, destaca-se a desinformação(11).

A participação ativa do adolescente nas discussões sobre sexualidade no ambiente escolar, inclusive o familiar é fundamental para que o cuidado a sua saúde seja de fato satisfatória.

Desse modo, toda Educação Sexual implica em reeducação da própria sexualidade. Os/as educadores/as sentem dificuldade pessoal em compreender a complexidade da sexualidade humana, por envolver posturas, tabus, crenças e valores a respeito de relacionamentos e comportamentos sexuais, requerendo olhar reflexivo sobre a própria sexualidade, dedicação e estudo ${ }^{(12)}$.

Entretanto, o desconhecimento ou o pouco conhecimento sobre o significado da sexualidade influencia negativamente os adolescentes a se exporem a riscos, pois as dúvidas sobre o assunto permanecem, e com o advento da internet, o diálogo aberto muitas vezes deixa de existir.

Muitas vezes a prática sexual na adolescência é realizada sem proteção, o que possibilita a vivência de riscos que podem resultar em uma série de transtornos, como contrair doenças sexualmente transmissiveis (DST) e ter uma gravidez indesejada, decorrentes da imaturidade na escotha de métodos contraceptivos e da falta de informações e discussões sobre sexualidade e anticon-cepção(2).

Nesse sentido, é fundamental que os adolescentes tenham acesso à informação de boa qualidade, para que se conscientizem dos benefícios e riscos a que estão expostos caso não utilizem os métodos contraceptivos de forma adequada. Estudos evidenciam que a família, a escola e a sociedade têm papéis importantes na vida dos jovens, pois são eles que têm a missão de desenvolver ações educativas, para que esses possam vivenciar sua sexualidade de uma maneira segura e responsável(2).

\section{Limitações do estudo}

Falar de sexualidade em pleno século XXI ainda encontra muitas barreiras, pois para a abordagem do assunto percebem-se limitações quanto à formação do vínculo para discutir o tema, em especial relacionado ao olhar do responsável frente às questões biológicas, psicossociais, in- clusive jurídicas envolvendo adolescentes. Estes estão em processo de mudanças comportamentais e sociais e tem ainda o complicador do seio familiar devido ao olhar não permissivo, muitas vezes reprimido para discutir o tema na escola, e muitas vezes na família.

Frente a isso, ainda como limitação inclui-se o fato de alguns dos responsáveis pelos adolescentes não terem comparecido à escola para ciência desta pesquisa em saúde. Assim, alguns foram excluídos da população, pois não havia responsável para aceitar e assinar o Termo de Consentimento Livre e Esclarecido. Na pesquisa não houve maiores dificuldades, apenas no aspecto sentimental (timidez) por dois membros por alguns minutos, superado no decorrer da entrevista.

\section{Contribuições do estudo para a prática}

A contribuição desta pesquisa é de extrema relevância para fomentar mais publicações relacionadas à promoção a saúde do adolescente na escola, especificamente sobre sexualidade. Tal importância está diretamente relacionada à necessidade da integração ensino, serviço e comunidade onde a saúde e a educação devem ser desenvolvidas de forma integral com a participação de todos os envolvidos, atendendo ao controle social como uma das diretrizes do Sistema Único de Saúde. No contexto deste estudo, a educação em saúde tem implicações diretas aos profissionais de saúde, principalmente para o enfermeiro que no seu atendimento deve desenvolver estratégias tecnológicas educacionais para orientar os adolescentes acerca de sua própria saúde, seja na escola seja nos serviços de saúde.

\section{CONSIDERAÇÕES FINAIS}

Estudos no Brasil vem abordando o tema sexualidade na adolescência com o objetivo principal de prevenir Infecções Sexualmente Transmissiveis (IST) e gravidez na adolescência. Vêm apontando o tema com certa discrição sobre o direito à vida sexual dos adolescentes, envolvendo pouco a família e a comunidade. Dessa forma investigações sobre o conhecimento acerca da sexualidade se fazem necessárias no contexto social em que o adolescente está inserido para assim contribuir no desenvolvimento de sua sexualidade.

A partir da pesquisa desenvolvida identificou-se que a maioria dos participantes desconhece o conceito sexualidade, demostrando pouco conhecimento acerca do tema evidenciando dificuldades para discutir o tema, devido alguns aspectos, como: vergonha, tensão, e medo, retraindo assim o adolescente de exercer seu direito à informação. A enfermagem possui uma importância peculiar para atuar 
nesses contextos, tanto no que se refere ao cuidado quanto na promoção da saúde.

Diante disso, a educação em saúde se desenvolve com práticas educativas voltadas à saúde sexual e para o exercício da sexualidade, orientando os(as) adolescentes quanto a seus direitos para que possam fazer escolhas saudáveis de acordo com seus projetos de vida e decidir, em que momento e se querem ou não, iniciar ou ter relações sexuais. Propõese, entre outros esforços a inclusão de enfermeiros escola- res na educação em saúde praticada nesses ambientes, envolvendo o corpo discente, docente e pais dos alunos.

\section{Contribuição do autores}

Concepção e/ou desenho, análise e interpretação dos dados, redação do artigo, revisão crítica, revisão final: Adriana Leal Moraes, Sandra Cristina dos Santos Costa, Selma Silva da Silva, Margarete Feio Boulhosa, Elisa da Silva Feitosa, Carla Monique Lavareda Costa.

\section{REFERÊNCIAS}

1. Ministério da Saúde (BR). Estatuto da criança e do adolescente: Lei n. 8.069, de 13 de julho de 1990, e legislação correlata. - 13. ed. (Série legislação; n. 175) - Brasília: Câmara dos Deputados, Edições Câmara, 2015. Available from: file:///C:/Users/JOAQUIM/Downloads/ estatuto_crianca_adolescente_13ed.pdf

2. Antunes, S. Conhecimento e comportamento dos adolescentes de uma escola pública sobre sexualidade e métodos contraceptivos. Revista Baiana de Saúde Pública, [S.l.], v. 41, n. 1, dez. 2017. Available from: <http://rbsp.sesab.ba.gov.br/index.php/rbsp/article/ view/2408>

3. Oliveira, RNG. Limites e possibilidades de um jogo online para a construção de conhecimento de adolescentes sobre a sexualidade. Ciênc. saúde coletiva, Rio de Janeiro, v. 21, n. 8, p. 2383-2392, ago. 2016. Available from: http://www.scielo.br/scielo.php?script=sci_ arttext\&pid=S1413-81232016000802383\&lng=pt\&nrm=iso

4. Ministério da Saúde (BR). Protocolo Clínico e Diretrizes Terapêuticas para Atenção Integral às Pessoas com Infecções Sexualmente Transmissiveis (IST) - Brasilia: Ministério da Saúde, 2019. 248 p.: il. Available from: http://www.aids.gov.br/pt-br/pub/2015/ protocolo-clinico-e-diretrizes-terapeuticas-para-atencao-integralpessoas-com-infeccoes

5. Ferreira, IT, Neves KTQ, Oliveira AWN, Galvão TRAF, Mangane, EM, Sousa LB. Avaliação da qualidade da consulta de enfermagem em infecções sexualmente transmissiveis. Enferm. Foco internet] 2018 [cited 2018; 9 (3): 42-47. Available from: http://revista.cofen.gov.br/ index.php/enfermagem/article/view/1119/459

6. Minayo, M. O desafio do conhecimento: pesquisa qualitativa em saúde. 10 ạ ed. Rio de Janeiro: Huctec-Abrasco, 2014
7. Soares ADAS, Rocha SS, Araújo Filho ACA. O cuidado com o crescimento e desenvolvimento infantil no discurso de pais e mães adolescentes. Enferm. Foco 2016 [cited 2016; 7 (3/4): 17-21 1]. Available from: https://www.researchgate.net/publication/314484688

8. Ibiapina LG. Assistência de enfermagem às adolescentes gestantes sob a ótica de callista roy. Enfermagem em Foco, [S.L.], v. 7, n. 3/4 p. 46-50, fev. 2017. Available from: http://revista.cofen.gov.br/index. php/enfermagem/article/view/915/345

9. Ministério da Saúde (BR). Cuidando de Adolescentes: orientações básicas para a saúde sexual e a saúde reprodutiva. - 2. ed. - Brasilia: Ministério da Saúde, 2018. 44 p. : il. Available from: http://bvsms.saude.gov.br/bvs/publicacoes/cuidando_adolescentes_saude_sexual_ reprodutiva_2ed.pdf

10. Araújo T, Monteiro C, Mesquita G, Alves E, Carvalho K, Monteiro R. Fatores de risco para infecção por HIV em adolescentes [Risk factors for HIV infection in adolescents] [Factores de riesgo para infección por VIH en adolescentes]. Revista Enfermagem UERJ, 20, out. 2012. Available from: http://www.e-publicacoes.uerj.br/index.php/ enfermagemuerj/article/view/4072

11. Rabin EG, Waterkemper R, Caregnato RC, \& de Souza, EN. Falando sobre sexualidade na adolescência: relato de experiência. Revista brasileira de extensão universitária, 2014, 5(1). 7-11. Available from: https://periodicos.uffs.edu.br/index.php/RBEU/article/view/895

12. Miranda JR. Educação sexual e sexualidades: reflexões sobre ações extensionistas na formação de professores/as. Extensio: R. Eletr. de Extensão, ISSN 1807-0221 Florianópolis, v. 15, n. 28, p. 168181, 2018. Available from: file:///C:/Users/Usuario/Downloads/51611190364-2-PB.pdf.

RECEBIDO EM: 02/01/2018

ACEITO EM: 21/02/2019.

154 I Enferm Foco [Internet]. 2019;10(2): 149-154. 